\title{
At the heart of mitochondrial quality control: many roads to the top
}

\author{
Roberta A. Gottlieb ${ }^{1} \cdot$ Honit Piplani ${ }^{1}$. Jon $\operatorname{Sin}^{1} \cdot$ Savannah Sawaged ${ }^{1} \cdot$ Syed M. Hamid ${ }^{1}$. David J. Taylor ${ }^{1}$. \\ Juliana de Freitas Germano ${ }^{1}$
}

Accepted: 8 January 2021 / Published online: 5 February 2021

(c) The Author(s) 2021

\begin{abstract}
Mitochondrial quality control depends upon selective elimination of damaged mitochondria, replacement by mitochondrial biogenesis, redistribution of mitochondrial components across the network by fusion, and segregation of damaged mitochondria by fission prior to mitophagy. In this review, we focus on mitochondrial dynamics (fusion/fission), mitophagy, and other mechanisms supporting mitochondrial quality control including maintenance of mtDNA and the mitochondrial unfolded protein response, particularly in the context of the heart.
\end{abstract}

Keywords Mitochondria $\cdot$ Mitophagy $\cdot$ Fusion $\cdot$ Fission $\cdot$ Cardiac

\section{Introduction}

Mitochondrial quality control plays a key role in long-lived cells including cardiomyocytes and neurons. Non-dividing cells require mechanisms to replace or repair proteins, complex assemblies of enzymatic machinery, and organelles, as well as membranes and potentially even DNA. In this review, we will focus on mitochondria, while acknowledging that repair/replacement mechanisms must also exist for other organelles including peroxisomes, lysosomes, endoplasmic reticulum, etc. While mitochondria are made up of protein assemblies, many of these are embedded in a lipid bilayer that requires ongoing maintenance, and uniquely, mitochondria are the only organelle with their own genome; thus, the integrity of mitochondrial DNA (mtDNA) must also be maintained. In this review, we will focus primarily on mitophagy. Mitochondrial quality control involves selective elimination of damaged mitochondria, replacement by mitochondrial biogenesis, redistributing newly imported proteins across the mitochondrial network by fusion, and segregation of damaged portions of the mitochondrial network by fission prior to mitophagy.

Roberta A. Gottlieb

Roberta.Gottlieb@cshs.org

Smidt Heart Institute, Cedars-Sinai Medical Center, AHSP9313, 127 S. San Vicente Blvd., Los Angeles, CA 90048, USA
When the heart is stressed, mitochondria are frequently observed to change size and shape. The processes that mediate the fusion, fission and fragmentation of mitochondria comprise mitochondrial dynamics, and a competent mitochondrial dynamics system is essential for embryonic survival [1-3]. In general, mitochondria are regarded as existing on a binary spectrum between two extremes of fusion and fission. The common designations 'elongated/tubular/fused' and 'fragmented/fissed' are typically employed to describe these relative morphologic states, though more sophisticated descriptions also exist which incorporate interconnectedness along with size. The functional consequences of a fused mitochondrial network compared with a fragmented one are poorly understood; however, much of what is known about these morphologic states relates to how they interact with the mitochondrial quality control machinery.

\section{Molecular regulation of mitochondrial dynamics}

The fusion/fission machinery is composed of dynamin-like GTPase proteins which reside either in the cytoplasm or on the inner (IMM) and outer mitochondrial membranes (OMM). Dynamin-related protein 1 (Drp1, or DNM1L), mainly located in the cytoplasm, is the principal effector of fission. Drp1 is activated by post-translational modifications and is recruited to mitochondria where it interacts with resident OMM receptors Mff, MiD49 and MiD51 [4]. Active 
Drp1 undergoes oligomerization, leading to membrane constriction with the process terminating with scission of the organelle mediated by Dynamin 2 [5]. Mitofusins 1 and 2 (Mfn1/2) are OMM resident GTPases, while Optic Atrophy 1 (Opa1) is located on the IMM. Collectively, they mediate mitochondrial fusion, though their regulation and function are incompletely understood. One reason for this discrepancy is that though a few target sites have been proposed, there are no established mechanisms of post-translational regulation of Mfn 1/2 or Opa1, with the exception of proteolytic processing of Opa1. Moreover, the distinction between fusion and fission apparatus is not cut and dried since although Opa1 is recognized as a fusion protein, it frequently participates in fragmentation when proteolytic degradation alters its fusion activity [6]. One striking feature of mitochondrial dynamics is that mitochondrial networks can rapidly transition from a fused to a fragmented state in response to stress, while conditions that promote mitochondrial fusion lead to much slower morphologic changes. Acute mitochondrial fragmentation is frequently observed under conditions of increased energy demands, following either physiological or pathological stimuli in the exercising or ischemic heart, respectively $[7,8]$. There are several theories which may explain why more active mitochondria undergo fragmentation. We have speculated that fragmentation may serve to increase surface area for nutrient and oxygen exchange, which will facilitate respiration-analogous to breaking up a long banquet table into smaller tables which increases the available seating. Alternatively, if membrane surface area is held constant during fragmentation, matrix volume will decrease, potentially elevating concentration of key solutes including $\mathrm{Ca}^{+2}$ (Fig. 1). Another intriguing hypothesis is that heat generated by active mitochondria poses a thermal stress to these organelles, and that by increasing the mitochondrial membrane surface area in contact with cytosol, this thermal energy can be more rapidly dissipated. Finally, there exists a large body of data to support a role for mitochondrial fission as a key step in the process of maintaining cellular

\footnotetext{
As an object increases in size, its volume increases as the cube of its linear dimensions while surface area increases as the square.

Assuming $\mathrm{Ca}^{++}$entry is directly related to surface area, $\mathrm{Ca}^{++}$ concentration will be lower in fused mitochondria.

Thus fused mitochondria will require more calcium to exceed the threshold for MPTP opening.

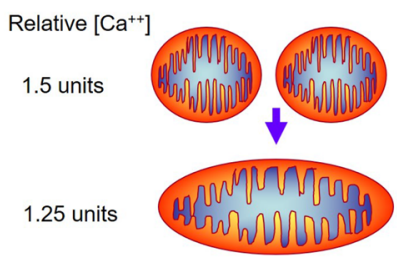

Conversely, mitochondria undergoing fission will have a higher $\mathrm{Ca}^{++}$concentration, which will increase electron transport chain activity and therefore ATP output—until $\left[\mathrm{Ca}^{++}\right]$exceeds the mPTP threshold.
}

Fig. 1 Surface area: volume relationship in mitochondria homeostasis through the sequestration and subsequent elimination of damaged mitochondria by mitophagy.

The OMM allows selective transport of metabolites and connects the mitochondria to neighboring organelles. The IMM contains two separate compartments, the intermembrane space and the matrix, that are uniquely characterized by their cristae structures. The cristae provide the sites of the respiratory chain that are stabilized by the phospholipid cardiolipin (CL) whose IMM binding is required for the function of the ADP-ATP translocator, supercomplex stability, and cytochrome c oxidase [9, 10]. Under mitochondrial dysfunction, however, the CL pool undergoes significant change to alter mitochondrial dynamics and trigger mitophagy. CL exhibits further structural roles within the IMM to increase mitochondrial size: genetic mutation of the cardiac and skeletal muscle tafazzin results in $80 \%$ deficiency of CL synthesis and metabolism which is thought to contribute to cardiomyopathy in Barth Syndrome patients [11]. CL deficiency is further observed to cause cristae disorganization within cardiomyocytes and fibroblasts, ultimately giving way to decreased CL biosynthesis and initiation of mitophagy $[12,13]$. In canonical mitophagy, CL serves as a signal for autophagic proteins to recognize dysfunctional mitochondria for degradation [14]. The mechanism of CL exposure from the IMM to OMM for cytosolic protein recognition remains in question, whether CL is translocated or perhaps externalized to the OMM during mitophagy.

One mechanism proposed for CL remodeling is the translocation of CL by phospholipid scramblases (PLS) that mediate translocation of phospholipids between the membrane bilayer. PLSCR3 is specifically enriched in the IMM and is important for maintenance of mitochondrial mass and respiration, yet is also required for sensitivity for cyt $c$ release and CL mobilization [15]. UV irradiation nearly doubles the amount of CL on the OMM in a model of WT vs. mutant PLSCR3 HEK293 cells, suggesting that PLSCR3 facilitates CL transport. Another IMM protein NDPK-D has recently been demonstrated to facilitate CL mobilization upon mitochondrial uncoupling with CCCP in murine lung epithelial cells and human HeLa cells [16]. Interestingly, Kagan et al. discovered a novel interaction of NDPK-D with OPA1, implicating a close relationship of fission-fusion in PLS-mediated CL translocation.

Another proposed mechanism of exposure of CL is through OMM pores formed by Bax/tBid interaction [17, 18]. Evidence for membrane permeabilization by these apoptosis family member proteins comes from a combination of studies using mutagenesis [19], surface plasmon resonance [20], and glycosylation mapping [21] to determine the precise membrane-binding interactions. Specifically, during apoptosis, proteolysis of Bid by caspase-8 [22] or calpain [23] generates the active truncated form of Bid (tBid). The processed tBid interacts with Bax to promote 
the formation of pore-like structures in the OMM, enabling cytochrome $c$ release from the IMM and potentially allowing cytosolic proteins to interact with CL on the inner membrane [24]. This can be amplified through a feedback cycle of CL interaction with procaspase- 8 to facilitate processing to caspase- 8 which can then process more Bid to tBid [25].

\section{Mitochondrial dynamics and mitochondrial quality control}

For damaged mitochondria to be removed from the cell, they must be engulfed by the autophagosomal double-membrane structure as the phagophore is elongating. Mitochondrial dynamics and mitochondrial quality control are intimately linked in the heart: Several mitochondrial membrane fusion/ fission proteins also interact with autophagy adaptors and effectors [26, 27]; furthermore, genetic ablation of fusion/ fission proteins frequently leads to disordered mitochondrial autophagy [28-30].

While a clear picture of the contributions of fusion and fission to mitochondrial quality control has yet to emerge, it is generally assumed that during these processes, there is a sorting of mitochondrial components which segregates damaged proteins and organellar components away from the rest of the mitochondrial network. The concept of asymmetric mitochondrial fission was first described by the Shirihai group in $\beta$ cells [31]. Dividing mitochondria were observed to exhibit different membrane potentials and those with higher membrane potentials were found to be more likely to fuse with other mitochondria. This of course begs the question of whether and how membrane potential could differ along the length of a mitochondrion prior to fission. That has recently been addressed by work [32] showing that individual mitochondrial cristae can have differing membrane potential; thus, it may be possible to have one region of a mitochondrion with high membrane potential (intact functioning individual cristae) and another region with low membrane potential (loss of cristae junctions and proton pumping capacity). After fission, mitochondria with lower membrane potential remained separate from the network and eventually are targeted for mitophagy, and thus, it was hypothesized that fission enables selection of mitochondria prior to autophagy. Healthy mitochondria would be capable of reintegration into the network, while damaged mitochondria would be unable to maintain adequate membrane potential and would subsequently be targeted for autophagic degradation. One mechanism for ensuring this is mediated by PINK1/Parkin, in which low membrane potential allows the accumulation of PINK1 on the outer membrane due to failure of protein import (which requires adequate membrane potential), and recruitment of Parkin, which ubiquitinates OM proteins. Ubiquitylation of Mfn1/Mfn2 prevents the mitochondrion from rejoining the network. It follows that during fusion, or in the interval between fission events, mitochondria sequester their healthy or damaged components to opposing poles of the mitochondria. Asymmetric fission of mitochondria has yet to be demonstrated in the intact heart, possibly in part due to the challenge of resolving mitochondria in adult cardiomyocytes using live microscopic techniques. Mitochondrial dynamism may operate differently in the heart than the evidence from cultured models suggests; some investigators have questioned the existence of networks of mitochondria in cardiac myocytes [33]. Recently however, Glancy et al. employed focused ion beam scanning electron microscopy to generate a three-dimensional model of cardiac mitochondria [34]. Their model proposed that multiple sub-networks of mitochondria exist in a cardiomyocyte, connected to the larger network via specific intermitochondrial junctions. Using live imaging, the authors further proposed that physical separation from the network occurs in malfunctioning mitochondria, leading to retraction of elongated mitochondria into condensed structures. It is reasonable to hypothesize that these separating mitochondria are undergoing fission, and we might, therefore, predict that there is increased mitochondrial autophagy activity in the electrically separated sub-networks. Recently, mitochondrial fusion events were demonstrated for the first time in adult ventricular cardiomyocytes [35]. Interestingly mitochondrial fusion rates decreased rapidly in culture in association with a decrease in calcium transient-driven contractile activity. Here, electrical uncoupling of the cardiomyocyte appeared to result in loss of fusion activity and in the previously mentioned Glancy report, electrical uncoupling of mitochondrial sub-networks had the same effect-suggesting that sustained cardiomyocyte-mitochondrial electrical coupling is important for maintaining mitochondrial fusion activity.

While there is evidence in cell models that piecemeal or bit-by-bit mitophagy is able to effectively sequester and degrade a portion of a mitochondrion, leaving the remaining organelle intact [36], it appears that the process of mitochondrial autophagy is generally impaired when mitochondria are more fused. In adult hearts lacking Drp1, mitochondria were elongated and dysfunctional [29]. Our unpublished data affirm this finding: dominant negative Drp1 suppressed basal autophagy and that induced by simulated ischemia/ reperfusion (sI/R) in HL-1 cells, while wild-type Drp1 overexpression increased autophagy and decreased apoptosis in response to sI/R injury [Anne Hamacher-Brady and Roberta Gottlieb, unpublished data]. In Drp1-null hearts, mitochondria-associated p62 was increased, suggesting that while the initiation of mitophagy was enhanced, autophagic flux was impaired, and further in vitro studies using shRNA to silence Drp1 indicated that mitochondrial translocation to lysosomes was impaired even under basal conditions [29]. It is intuitive that there will be a greater cost to the cell 
to manufacture an autophagosome to encircle and engulf a larger fused mitochondrion than a smaller one; however, the relationship between fission and autophagy is likely more complex.

In addition to consideration of fission, it is important to understand the importance of fusion in mitochondrial quality control. Global constitutive knockout of both mitofusins results in embryonic lethality [3]. When both mitofusins are conditionally deleted in the heart, fusion activity is ablated, mitochondria appear smaller, and exhibit impaired rates of oxygen consumption [37]. Disruption of mitochondrial fusion in skeletal muscle through conditional deletion of Mfn1/2 (MLC1f promoter) results in accumulation of mtDNA point mutations and deletions and mtDNA depletion, resulting in muscle atrophy and impaired function [38]. Interestingly, the resulting energy deficit is accompanied by proliferation of small mitochondria with profoundly reduced mtDNA content ( 250 copies of mtDNA per nuclear genome in double $\mathrm{KO}$ vs 3500 copies in wild-type). Cardiacrestricted deletion of $\mathrm{Mfn} 1 / 2$ results in a similar proliferation of abnormal-appearing mitochondria, severe cardiomyopathy, and death by postnatal day 16 [39]. Hearts of these mice also exhibited mtDNA depletion. Interestingly, inducible cardiac-restricted deletion of Mfn1/2 exhibited mitochondrial dysfunction and poor cardiac contractility but a reduction in infarct size after acute I/R injury [40]. While disruption of fusion or fission individually is associated with significant mitochondrial dysfunction, the combined deletion of Mfn1/Mfn2 and Drp1 in the heart results in longer survival than either Mfn1/2 DKO or the Drp1 KO, but the mice eventually develop a unique hypertrophy associated with accumulation of mitochondria, impaired mitophagy, and mtDNA depletion [28].

\section{Canonical mitophagy}

The best-characterized process for mitophagy is accomplished through the actions of PINK1/Parkin/P62. The genes encoding PINK1 and Parkin are strongly implicated as mediators of familial and sporadic Parkinson's disease [41], and the first studies showing the interaction between these proteins in vivo identified a role of Parkin in restoring mitochondrial morphology and function downstream of PINK1 in mutant models of Drosophila melanogaster [42-44]. Further investigations confirmed PINK1-Parkin interactions in mammalian cells [45]. In this well-studied process, PINK1 is constitutively imported into the mitochondrion, where it is degraded by PARL in the intermembrane space [46] or Lon protease (LonP1) in the matrix [47].In the absence of adequate mitochondrial membrane potential PINK1 kinase is stabilized on the outer mitochondrial membrane [48]. As PINK1 accumulates, it phosphorylates multiple protein targets including ubiquitin $[49,50][50,51]$; phosphoubiquitin activates and recruits Parkin, a cytosolic E3 ubiquitin ligase [50-54]. A recent study demonstrated that PINK1 also has a direct role in Parkin phosphorylation and subsequent activation [55]. Following Parkin activation and translocation, cytosolic p62 translocates to the mitochondria and binds to the polymerized ubiquitin through its ubiquitin-binding domain, and works as an adaptor connecting the damaged mitochondria to membrane of the autophagosomes through its LC3-interacting region (LIR), contributing to mitophagy completion [56]. PINK1 phosphorylation of ubiquitin and other targets can recruit NDP52 and optineurin to initiate Parkin-independent (but ubiquitindependent) mitophagy [57].

Optineurin, first isolated by yeast two hybrid screening in 1998 , is a $67-K D a$ protein implicated in many inflammatory conditions including cardiac ischemic disease. Optineurin is known to be involved in multiple processes of the cell such as autophagy [58], cell division [59], protein trafficking [60] and inflammatory signaling [61]. Optineurin can regulate mitophagy both through ubiquitin-dependent and -independent mechanisms. Optineurin interacts with LC3 through its LIR domain and this interaction is facilitated with phosphorylation by TBK1 at Ser177 [58]. In response to mitochondrial depolarization, TBK1 undergoes activating phosphorylation in a Parkin-PINK1 dependent manner [62], which facilitates optineurin recruitment to the damaged mitochondrion [57]. Following recruitment, optineurin binds with polyubiquitin chain through its Ub-binding domain (UBD) in ABIN proteins and NEMO (UBAN). Active TBK1 also phosphorylates optineurin at Ser473 and Ser513, stimulating the latter's interaction with ubiquitin [63]. Optineurin also induces autophagosome formation by recruiting autophagy-associated proteins, namely the Atg 125-16L1 complex [64]. In the ubiquitin-independent mechanism of optineurin, LC3 family members play an important role by recruiting optineurin to mitochondria by ubiquitinlike Atg8 protein. This further leads to a positive feedback loop mechanism of Atg8 lipidation [65]. We also recently documented the importance of optineurin in hypothermiamediated cardioprotection [66]. Here, we showed that myocardial hypothermia applied after ischemia and reperfusion activated mitophagy and enhanced autophagic flux, reflected by downregulation of mitophagy markers including optineurin, parkin, and polyubiquitin chains in heavy membrane fraction. This event was accompanied by increased short form OPA1, MFF and DRP1. This process is summarized in Fig. 2.

Because the heart is a high-energy demanding organ, high-efficiency mitochondrial quality control preserves the functionality of the cardiomyocytes, and one would expect that PINK1/Parkin/p62 pathway would be involved in this regulation. Further experiments 


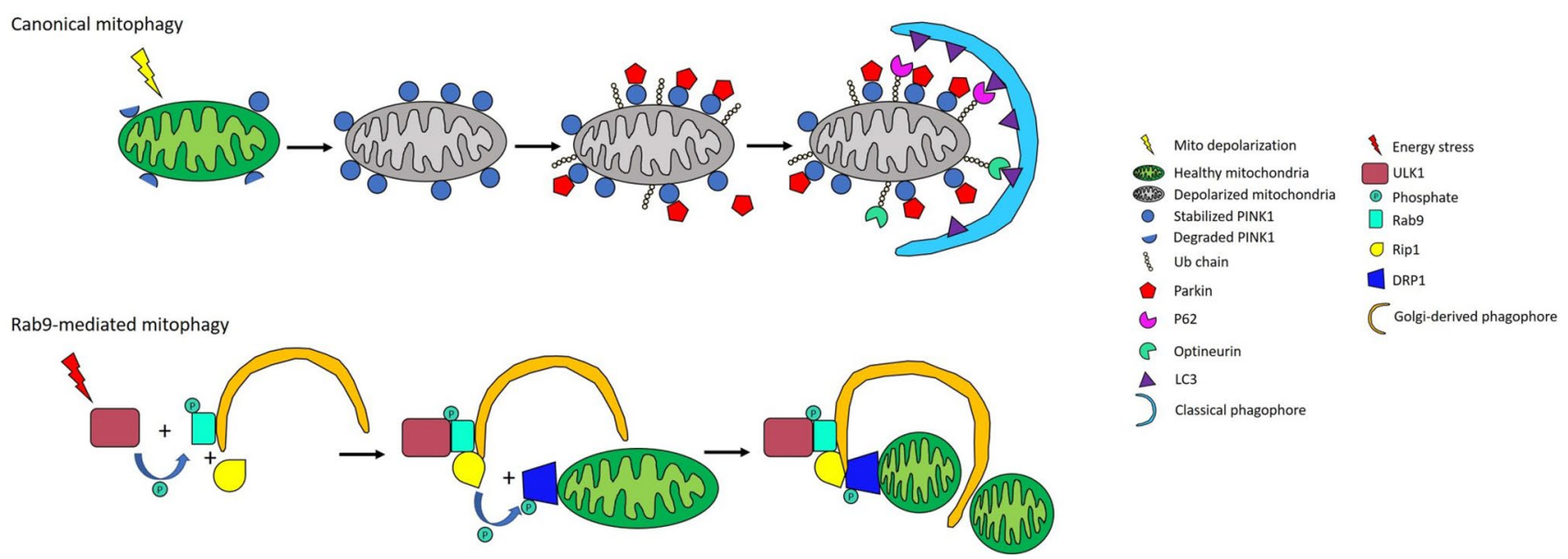

Fig. 2 Canonical and alternative mitophagy pathways

in mouse embryonic cardiomyocytes and fibroblasts revealed that Mfn2, an outer membrane fusion protein, gets phosphorylated by PINK1 and functions as a Parkin receptor [27]. Despite the existence of studies showing that Parkin-mediated mitophagy is dispensable for basal mitophagy in tissues of high metabolic demand, including the heart [67], further in vivo experiments supported the in vitro investigations regarding the role of Parkin in mitophagy. More specifically in cardiac disease, it was observed that Parkin accumulated in the border zone of wild-type infarcted mice but not in their Parkinknockout (KO) counterparts, suggesting that Parkin is essential for mitophagy activation under myocardial stress [68]. Cardiac hypertrophy was also identified as a consequence of PINK1 knockout in mice, and PINK1 protein is drastically decreased in heart failure [69]. Further experiments revealed that cardiac hypertrophy as a consequence of diabetic cardiomyopathy induced by a high-fat diet was attenuated by but not exclusive of Parkin-mediated mitophagy, accompanied by attenuation of ventricular diastolic dysfunction [70]. Our group specifically demonstrated for the first time the critical role of Parkin/p62-mediated mitophagy in cardioprotection in an ischemic preconditioning model in mouse hearts [71]; subsequently, this mechanism was also validated in human atrial tissue during cardiopulmonary bypass heart surgery [72]. No evidence was found for the participation of Parkin in the clearance of mitochondria with damaged mtDNA in the cardiac aging process, as showed by Woodall et al. [73]. However, not only Parkin is essential for mitochondrial homeostasis during cardiac stress conditions but also it was found to be crucial for mitochondrial plasticity and metabolic reprogramming from carbohydrates to fatty acid oxidation in the perinatal mouse heart [74]. Moreover, Parkin was also implicated in the regulation of mitochondrial biogenesis in neurons through interaction with PGC- $1 \alpha$ and ubiquitination of PINK1-phosphorylated PARIS (Parkin Interacting Substrate), which has a role in repressing PGC- $1 \alpha$. This mechanism, however, has not yet been analyzed in the heart. All these findings suggest that Parkin is a multifunctional protein with an essential role in maintaining mitochondrial quality control in different tissues through diverse mechanisms such as mitophagy, mitochondrial metabolic reprogramming and mitochondrial biogenesis.

A study by Hood's group of age-related deterioration of skeletal muscle showed that exercise-induced mitophagy was mediated by Parkin, and that with aging, Parkin levels increased while mitochondrial respiration decreased [75]. The impaired respiration was worse in Parkin KO mice and was accompanied by increased ROS production in aged Parkin KO mice. Despite elevated Parkin protein in aged mice, its translocation to mitochondria after exercise was attenuated, in contrast to increased translocation in young mice. They also observed an inverse relationship between PGC- $1 \alpha$ and PARIS, the repressor.

\section{Ubiquitin-independent mitophagy}

In ubiquitin-independent mitophagy, autophagy receptors bind directly to dysfunctional mitochondria through the proteins present on the OMM, which link the dysfunctional mitochondria to autophagosomes. These receptors include Bnip3 (Bcl-2/adenovirus E1B interacting protein 3) and FUNDC1 present on OMM. These receptors 
contain one or more LIR (LC3-interacting region) [76] which bind to LC3 or GABARAP (gamma-aminobutyric acid receptor-associated protein) present on the phagophore. This interaction is regulated by phosphorylation status of the receptors; for example, Bnip3 phosphorylation on Serine residues 17 and 24 enhances binding to LC3B and GATE-16 [77]. Bnip3 is a hypoxia-inducible protein and a member of the proapoptotic Bcl-2 family, possessing a $\mathrm{BH} 3$ domain. Bnip3 integrates into the OMM via a carboxy terminal transmembrane (TM) domain and interacts with LC3 through the amino terminal domain [78]. Bnip3 was initially reported to induce cell death [79] through outer mitochondrial membrane permeabilization and cytochrome $c$ release [80] as well as triggering the mitochondrial permeability transition pore (mPTP). Increasing evidence also implicate its role in cell survival by inducing autophagy $[81,82]$ and competition with Beclin1 for Bcl-2/Bcl-xL. Our group showed that Bnip3-mediated autophagy was mPTP independent [83]. Stress such as hypoxia induces mitochondrial quality by mitophagy through Bnip3 [82, 84]. Bnip3's dual roles in cell fate suggest it is a pivotal regulator in disease processes [85, 86]. The Bnip 3 promoter contains a consensus sequence for HIF- $1 \alpha$ binding where it acts as transcription factor to drive Bnip3 expression [87]. One of the mechanisms shown to restrict Bnip3 expression in hypoxic conditions is methylation in the promoter region [88] which decreases the interaction between HIF- $1 \alpha$ and the Bnip3 promoter. Chaanine et al. (2013) showed that Bnip3 knockdown prevented apoptosis, fibrosis, adverse cardiac remodeling and improved diastolic and systolic function in a heart failure model [89]. Overexpression of Bnip3 induces mitochondrial fragmentation in cardiomyocytes. Bnip3 has also been shown to interact with VDAC resulting in oligomerization of VDAC and mitochondrial dysfunction [89]. However, induced overexpression of Bnip3 did not lead to cardiomyocyte apoptosis in neonatal mice hearts [90]. Increasing reports support Bnip3's dual roles to induce apoptosis and mitophagy; while phosphorylation may be one mechanism, other as yet unidentified processes may also regulate Bnip3's prosurvival/proapoptotic functions.

Another mitophagy adapter protein, FUNDC1, is also shown to interact with LC3 to induce mitophagy in response to hypoxia [91]. FUNDC1 maintains the mitochondria-ER contact sites and promotes mitochondrial calcium uptake [92]. FUNDC1 interaction with LC3 is enhanced by phosphorylation at serine 17 by ULK1; mitochondrial phosphoglycerate mutase, PGAM5, dephosphorylates FUNDC1 [93, 94]. FUNDC1 knockout mice showed cardiac dysfunction and increased mitochondrial fission and cell death [95]. FUNDC1 interacts with Opa1, and its dephosphorylation promotes dissociation, leading to mitochondrial fission via interaction with DNM1L and culminating with mitophagy
[96]. Many studies suggest the importance of FUNDC1 in regulating mitochondrial quality control and cardiac injury [97-99]; however, the detailed mechanism and interacting partners of FUNDC1 remain to be elucidated.

As a highly energetic organ, the heart heavily relies on properly functioning mitochondria to maintain normal function. Cardiac insults such as ischemia not only lead to mitochondrial dysfunction, but also excessive reactive oxygen species production and cell death. As such, the heart has been shown to be equipped with a number of alternate modes of mitochondrial clearance. Aside from the more traditional and well-characterized PINK/Parkin-mediated mitophagy pathway, another mechanism was recently identified revolving around Ulk1, Rab9, Rip1 and Drp1. Saito et al. reported that in a mouse model of coronary artery ligation, mitophagy was briskly activated in the heart [100]. Interestingly, mice with ATG7 knocked-out maintained this elevation in mitophagy; whereas, mitophagy was blunted when Ulk1 was knocked out. In this setting, Ulk1 appeared to play a predominant role in mediating mitophagy and expectedly, the absence of Ulk1 also corresponded with increased infarct size. They went on to show that these Ulk1-mediated autophagosomes originate from previously reported trans Golgi membranes enriched with Rab9 rather than LC3. Indeed, inhibiting Golgi membranes with brefeldin A revealed that mitophagy mediated by Ulk1 and Rab9 was impaired by brefeldin A, whereas conventional Parkin-mediated autophagy was unaltered by the drug. The authors showed that Rip1 induced the activating phosphorylation of Drp1 at S616 which triggered mitochondrial fission. Ulk1 was suggested to phosphorylate Rab9 at S179 which supported Rab9 and Rip1 interactions resulting in Drp1 phosphorylation. The authors suggest that these four proteins form a complex which indicates how mitochondrial fragments become trafficked into Ulk1-mediated autophagosomes (Fig. 2). This pathway appears to be important for cardiac homeostasis in diabetic cardiomyopathy [70], wherein Parkin is downregulated [101].

One might wonder why multiple mitophagy pathways may exist in the heart. Because the heart is highly reliant on healthy high functioning mitochondria, mitochondrial quality control machinery is crucial for maintaining cellular homeostasis. Therefore, mammals may have developed multiple context-dependent but only partially redundant mitophagy mechanisms.

\section{Quality control of the mitochondrial genome}

Disruption of mitochondrial dynamics consistently impacted mtDNA integrity; it is, therefore, essential to consider mtDNA in any discussion of mitochondrial quality control. As mtDNA is continuously exposed to reactive oxygen 
species as a byproduct of respiration (about $1-2 \%$ of electrons go to superoxide generation [102]), and because it is not shielded by histones, it is vulnerable to damage, making ongoing maintenance or culling of deleterious mtDNA an essential element of cellular homeostasis. Mitochondria contain multiple copies of circular double-stranded DNA (mtDNA), which encodes tRNAs (22), rRNAs (2), and polypeptides (13) essential for oxidative phosphorylation. As almost the entire sequence encodes proteins or structural RNAs, a mutation anywhere in the $\sim 16,000$-bp sequence is likely to have consequences. mtDNA is not encased in histones but is condensed into nucleoids with TFAM, which also plays a role in mtDNA replication and transcription [103].

mtDNA repair depends on polymerase gamma (which also is responsible for mtDNA replication) and 8-oxoguanine DNA glycosylase (mOGG1). Defects in mtDNA repair lead to multiple disorders including heart failure [104]. Recently, the DNA repair nuclease MRE11A was linked to mitochondrial dysfunction including release of mtDNA into the cytosol where it triggered inflammasome activation and pyroptosis [105]. Another enzyme, DNA2, which functions in removal of single strand DNA during mtDNA replication or Long Patch Base Excision Repair pathway, has been linked to familial and sporadic forms of mitochondrial myopathy [106]. Doubtless many other enzymes can affect mtDNA integrity and repair, and their impaired function may eventually culminate in heart failure. Defects in mtDNA maintenance have been reviewed recently [107].

The mitophagy/mito-biogenesis/fusion/fission program has been suggested to be able to compensate for mtDNA damage that results in impaired respiratory function, based on elegant mathematical modeling of the process [108]. A key aspect was the requirement for excluding depolarized mitochondria from participation in fusion events. Parkin ubiquitinates multiple outer membrane proteins including mitofusin 1 and 2 [109, 110], causing their degradation by the proteasomal system, thereby preventing subsequent fusion events, and simultaneously promoting mitophagy. With aging, mtDNA damage increases, but it is not completely established whether this is due to attenuated mitophagy (which decreases with age).

\section{Mitochondrial unfolded protein response}

Proper folding of proteins is essential for cellular homeostasis as aggregation of newly synthesized or imported misfolded proteins. Protein misfolding results in loss of individual protein functionality but also affects multi-protein complexes, leading to deleterious consequences for the cell [111]. To efficiently regulate protein folding processes, cells have developed distinct but highly integrated quality control mechanisms in the cytosol, endoplasmic reticulum and mitochondria. Cytosolic response to misfolded proteins relies heavily on a battery of heat shock proteins (Hsp), especially Hsp70, which leads to reprogramming of the cellular transcription program [112]. Endoplasmic reticulum has three highly conserved regulators of the unfolded response which provide surveillance across the ER membrane. These regulators-IRE1, PERK and ATF6-are kept dormant by the binding of Bip in the absence of misfolded proteins. Detachment of Bip due to accumulation of unfolded proteins leads to the activation of ER unfolded protein response which not only rewires the transcriptional program to increase the folding capacity but also suppresses RNA translation and decreases protein degradation programs to decrease the folding load [113, 114].

Regulation of unfolded proteins in mitochondria poses further challenges, primarily because of distinct structural compartmentalization and secondarily since mitochondrial proteins derive from both nuclear and mitochondrial genomes. Subunits translated from nuclear and mitochondrial transcripts must be assembled into the larger oxphos complexes in proper stoichiometry. Any imbalance between nuclear and mitochondrial protein synthesis can result in the accumulation of unincorporated proteins that may aggregate. The mitochondrial unfolded response UPR ${ }^{\mathrm{mt}}$ is a transcriptional stress response that is activated by multiple forms of mitochondrial dysfunction in any of these compartments and reprograms mitochondrial to nuclear communication [115, 116].

In worms, activating transcription factor 1 (ATFS-1) is a well-studied transcription factor that acts as a first responder of $\mathrm{UPR}^{\mathrm{mt}}$ activation. In addition to a mitochondrial targeting sequence (MTS), it also has a nuclear localization signal (NLS). Under homeostatic conditions, ATFS-1 is imported into mitochondria where it is degraded by LON protease $[117,118]$. When mitochondria are damaged, ATFS-1 is preferentially accumulated in nucleus and activates UPR ${ }^{\mathrm{mt}}$. Thus, compartmentalization of ATFS- 1 regulates its transcriptional activity, indicating that mitochondrial import machinery plays an important role in UPR ${ }^{\mathrm{mt}}$ induction. Although there are similarities in UPR ${ }^{\mathrm{mt}}$ activation between worms and mammals, this process is certainly more complex in mammals. Many studies have shown that key components of integrated stress response (ISR), namely CHOP, ATF4 and ATF5 are not only activated by multiple forms of mitochondrial stress but are also required for the proper induction of UPR ${ }^{\mathrm{mt}}[119,120]$. Among these, ATF5 is of special interest as it has been proposed to be a mammalian ortholog of ATFS-1 based on the fact that it can rescue UPR ${ }^{\mathrm{mt}}$ activation in the absence of ATFS-1 [121]. Moreover, ATF5 bears a mitochondrial targeting sequence (MTS) and its activity seems to be regulated by mitochondrial import like ATFS-1 [121]. Despite the fact that ISR effectors like CHOP and 
ATF4 are activated in canonical UPR ${ }^{\mathrm{mt}}$ signaling, the action of these factors is highly specific as they do not induce Bip, an ER chaperone important in UPR ${ }^{\mathrm{ER}}$ [119]. Activation of the targeted transcription program due to these transcription factors leads to an increase in mitochondrial chaperones, thereby increasing mitochondrial folding capacity.

Reduction of protein import into mitochondria and a decrease in translation are also part of the $\mathrm{UPR}^{\mathrm{mt}}$, which have been well documented in C. elegans $[117,122]$. This phenomenon has also been reported recently in mammalian cells under acute induction of UPR ${ }^{\mathrm{mt}}$, resulting in degradation of MRP3 transcript and protein [120]. This translational aspect of the UPR ${ }^{\mathrm{mt}}$ may act locally on a single damaged mitochondrion, bypassing the need to cross any cellular threshold levels; this may represent the first line defense against mitochondrial damage [123]. Activation of $\mathrm{UPR}^{\mathrm{mt}}$ also leads to the activation of estrogen receptor alpha (ER $\alpha)$, which leads to proteasome activation to decrease the misfolded protein burden especially in the inter-membrane space (IMS) [124, 125]. This signaling also activates HTRA2, a protease in the intermembrane space (IMS), and nuclear respiratory factor 1 (NRF1), which is involved in mitochondrial biogenesis [126]. LON protease is a member of AAA + proteases (ATPases associated with a variety of cellular activities), a broad group of ATP dependent proteases implicated in $\mathrm{UPR}^{\mathrm{mt}}$ and responsible for the degradation of misfolded proteins in the mitochondrial matrix [127-129]. Recently, there have been a couple of reports relating the $\mathrm{UPR}^{\mathrm{ER}}$ to $\mathrm{UPR}^{\mathrm{mt}}$. A critical regulator of ER unfolded response, PERK was shown to regulate mitochondrial morphology promoting mitochondrial hyper-fusion and inhibiting severe mitochondrial fragmentation under conditions of stress [130]. In another study, activation of ISR under lipid stress was shown to upregulate LONP1 resulting in increased mitochondrial ROS and inflammasome activation in macrophages [131]. Future studies will reveal more exciting aspects of interorganelle communication especially between mitochondria and endoplasmic reticulum.

Maintenance of mitochondrial function is vital for cell survival and functioning. This becomes of even more importance in organs where energy demand is high and cellular turnover is low, key characteristics of cardiomyocytes. Alterations in mitochondrial function due to impairment of mitochondrial quality control mechanisms are among the major causes of cardiac senescence and aging [132]. Mitochondrial unfolded protein response provides a robust mechanism for mitochondrial quality control by improving the homeostasis and limiting the damage.

\section{Conclusion}

While mitochondrial quality control may be unimportant for "disposable" cells, it is indispensable for long-lived cells such as cardiomyocytes and neurons, where defects in mitochondrial quality control lead to functional deficits as seen in Parkin disease and many forms of heart failure. Multiple pathways exist that may represent housekeeping processes versus responses to different types of cellular stress. It is increasingly clear that maintaining mitochondrial quality control is essential to preserving cardiac function, for without good mitochondrial function, the energy to support contraction will be jeopardized. Interventions to target mitochondrial turnover are likely to enrich the therapeutic arsenal for heart disease.

Open Access This article is licensed under a Creative Commons Attribution 4.0 International License, which permits use, sharing, adaptation, distribution and reproduction in any medium or format, as long as you give appropriate credit to the original author(s) and the source, provide a link to the Creative Commons licence, and indicate if changes were made. The images or other third party material in this article are included in the article's Creative Commons licence, unless indicated otherwise in a credit line to the material. If material is not included in the article's Creative Commons licence and your intended use is not permitted by statutory regulation or exceeds the permitted use, you will need to obtain permission directly from the copyright holder. To view a copy of this licence, visit http://creativecommons.org/licenses/by/4.0/.

\section{References}

1. Ishihara $\mathrm{N}$ et al (2009) Mitochondrial fission factor Drp1 is essential for embryonic development and synapse formation in mice. Nat Cell Biol 11(8):958-966

2. Davies VJ et al (2007) Opa1 deficiency in a mouse model of autosomal dominant optic atrophy impairs mitochondrial morphology, optic nerve structure and visual function. Hum Mol Genet 16(11):1307-1318

3. Chen $\mathrm{H}$ et al (2003) Mitofusins Mfn1 and Mfn2 coordinately regulate mitochondrial fusion and are essential for embryonic development. J Cell Biol 160(2):189-200

4. Nan J et al (2017) Molecular regulation of mitochondrial dynamics in cardiac disease. Biochim Biophys Acta Mol Cell Res 7:1260-1273

5. Lee JE et al (2016) Multiple dynamin family members collaborate to drive mitochondrial division. Nature 540:139

6. Lee H, Yoon Y (2018) Mitochondrial membrane dynamicsfunctional positioning of OPA1. Antioxidants (Basel, Switzerland) 7(12):186

7. Coronado $\mathrm{M}$ et al (2018) Physiological mitochondrial fragmentation is a normal cardiac adaptation to increased energy demand. Circ Res 122(2):282-295

8. Sharp WW et al (2014) Dynamin-related protein 1 (Drp1)mediated diastolic dysfunction in myocardial ischemia-reperfusion injury: therapeutic benefits of Drp1 inhibition to reduce mitochondrial fission. FASEB J 28(1):316-326

9. Pfeiffer $\mathrm{K}$ et al (2003) Cardiolipin stabilizes respiratory chain supercomplexes. J Biol Chem 278(52):52873-52880 
10. Jiang F et al (2000) Absence of cardiolipin in the crd1 null mutant results in decreased mitochondrial membrane potential and reduced mitochondrial function. J Biol Chem 275(29):22387-22394

11. $\mathrm{Xu} \mathrm{Y}$ et al (2006) A drosophila model of barth syndrome. Proc Natl Acad Sci U S A 103(31):11584-11588

12. Richter-Dennerlein R et al (2014) DNAJC19, a mitochondrial cochaperone associated with cardiomyopathy, forms a complex with prohibitins to regulate cardiolipin remodeling. Cell Metab 20(1):158-171

13. Hsu P et al (2015) Cardiolipin remodeling by TAZ/tafazzin is selectively required for the initiation of mitophagy. Autophagy 11(4):643-652

14. Chu CT et al (2013) Cardiolipin externalization to the outer mitochondrial membrane acts as an elimination signal for mitophagy in neuronal cells. Nat Cell Biol 15(10):1197-1205

15. Liu J et al (2003) Phospholipid scramblase 3 controls mitochondrial structure, function, and apoptotic response $11 \mathrm{NIH}$ grants K08CA795093 (RML) and K08AR48618 (DG) Huntsman Cancer Foundation. Mol Cancer Res 1(12):892

16. Kagan VE et al (2016) NDPK-D (NM23-H4)-mediated externalization of cardiolipin enables elimination of depolarized mitochondria by mitophagy. Cell Death Differ 23(7):1140-1151

17. Lovell JF et al (2008) Membrane binding by tBid initiates an ordered series of events culminating in membrane permeabilization by Bax. Cell 135(6):1074-1084

18. Korytowski W et al (2011) Permeabilization of the mitochondrial outer membrane by Bax/truncated Bid (tBid) proteins as sensitized by cardiolipin hydroperoxide translocation: mechanistic implications for the intrinsic pathway of oxidative apoptosis. J Biol Chem 286(30):26334-26343

19. Wang K et al (1996) BID: a novel BH3 domain-only death agonist. Genes Dev 10(22):2859-2869

20. Kim M et al (2005) Detection of Bax protein conformational change using a surface plasmon resonance imaging-based antibody chip. Biochem Biophys Res Commun 338(4):1834-1838

21. García-Sáez AJ et al (2004) Membrane-insertion fragments of Bcl-xL, Bax, and Bid. Biochemistry 43(34):10930-10943

22. Li H et al (1998) Cleavage of BID by caspase 8 mediates the mitochondrial damage in the Fas pathway of apoptosis. Cell 94(4):491-501

23. Chen $\mathrm{M}$ et al (2001) Bid is cleaved by calpain to an active fragment in vitro and during myocardial ischemia/reperfusion. J Biol Chem 276(33):30724-30728

24. Korsmeyer SJ et al (2000) Pro-apoptotic cascade activates BID, which oligomerizes BAK or BAX into pores that result in the release of cytochrome c. Cell Death Differ 7(12):1166-1173

25. Schug ZT et al (2011) BID is cleaved by caspase- 8 within a native complex on the mitochondrial membrane. Cell Death Differ 18(3):538-548

26. Gao J, Qin S, Jiang CA (2015) Parkin-induced ubiquitination of Mff promotes its association with p62/SQSTM1 during mitochondrial depolarization. Acta Biochim Biophys Sin 47(7):522-529

27. Chen Y, Dorn GW 2nd (2013) PINK1-phosphorylated mitofusin 2 is a Parkin receptor for culling damaged mitochondria. Science 340(6131):471-475

28. Song M et al (2017) Abrogating mitochondrial dynamics in mouse hearts accelerates mitochondrial senescence. Cell Metab 26(6):872-883.e5

29. Ikeda $Y$ et al (2015) Endogenous Drp1 mediates mitochondrial autophagy and protects the heart against energy stress. Circ Res 116(2):264-278
30. Song M et al (2015) Mitochondrial fission and fusion factors reciprocally orchestrate mitophagic culling in mouse hearts and cultured fibroblasts. Cell Metab 21(2):273-286

31. Twig G et al (2008) Fission and selective fusion govern mitochondrial segregation and elimination by autophagy. EMBO J 27(2):433-446

32. Wolf DM et al (2019) Individual cristae within the same mitochondrion display different membrane potentials and are functionally independent. EMBO J 38:e101056

33. Dorn GW 2nd (2018) Cardiac-specific research platforms engender novel insights into mitochondrial dynamism. Curr Opin Physiol 3:110-115

34. Glancy B et al (2017) Power grid protection of the muscle mitochondrial reticulum. Cell Rep 19(3):487-496

35. Eisner V et al (2017) Mitochondrial fusion dynamics is robust in the heart and depends on calcium oscillations and contractile activity. Proc Natl Acad Sci U S A 114(5):E859

36. Yang J-Y, Yang WY (2013) Bit-by-bit autophagic removal of parkin-labelled mitochondria. Nat Commun 4(1):2428

37. Chen Y, Liu Y, Dorn GW 2nd (2011) Mitochondrial fusion is essential for organelle function and cardiac homeostasis. Circ Res 109(12):1327-1331

38. Chen $\mathrm{H}$ et al (2010) Mitochondrial fusion is required for mtDNA stability in skeletal muscle and tolerance of mtDNA mutations. Cell 141(2):280-289

39. Papanicolaou KN et al (2012) Mitofusins 1 and 2 are essential for postnatal metabolic remodeling in heart. Circ Res 111(8):1012-1026

40. Hall AR et al (2016) Hearts deficient in both Mfn1 and Mfn2 are protected against acute myocardial infarction. Cell Death Dis 7:e2238

41. Dawson TM, Dawson VL (2010) The role of parkin in familial and sporadic Parkinson's disease. Mov Disord 25(Suppl 1):S32-S39

42. Yang Y et al (2006) Mitochondrial pathology and muscle and dopaminergic neuron degeneration caused by inactivation of Drosophila Pink1 is rescued by Parkin. Proc Natl Acad Sci U S A 103(28):10793-10798

43. Clark IE et al (2006) Drosophila pink1 is required for mitochondrial function and interacts genetically with parkin. Nature 441(7097):1162-1166

44. Park J et al (2006) Mitochondrial dysfunction in Drosophila PINK1 mutants is complemented by parkin. Nature 441(7097):1157-1161

45. Exner $\mathrm{N}$ et al (2007) Loss-of-function of human PINK1 results in mitochondrial pathology and can be rescued by parkin. J Neurosci 27(45):12413-12418

46. Jin SM et al (2010) Mitochondrial membrane potential regulates PINK1 import and proteolytic destabilization by PARL. J Cell Biol 191(5):933-942

47. Zurita Rendon O, Shoubridge EA (2018) LONP1 is required for maturation of a subset of mitochondrial proteins, and its loss elicits an integrated stress response. Mol Cell Biol 38(20):e00412-417

48. Narendra DP et al (2010) PINK1 is selectively stabilized on impaired mitochondria to activate Parkin. PLoS Biol 8(1):e1000298

49. Narendra D, Walker JE, Youle R (2012) Mitochondrial quality control mediated by PINK1 and Parkin: links to parkinsonism. Cold Spring Harb Perspect Biol 4(11):a011338

50. Kane LA et al (2014) PINK1 phosphorylates ubiquitin to activate Parkin E3 ubiquitin ligase activity. J Cell Biol 205(2):143-153

51. Koyano F et al (2014) Ubiquitin is phosphorylated by PINK1 to activate parkin. Nature 510(7503):162-166 
52. Iguchi M et al (2013) Parkin-catalyzed ubiquitin-ester transfer is triggered by PINK1-dependent phosphorylation. J Biol Chem 288(30):22019-22032

53. Kazlauskaite A et al (2014) Phosphorylation of Parkin at Serine65 is essential for activation: elaboration of a Miro1 substrate-based assay of Parkin E3 ligase activity. Open Biol 4:130213

54. Kazlauskaite A et al (2014) Parkin is activated by PINK1dependent phosphorylation of ubiquitin at Ser65. Biochem J 460(1):127-139

55. McWilliams TG et al (2018) Phosphorylation of Parkin at serine 65 is essential for its activation. Open Biol 8(11):180108

56. Geisler S et al (2010) PINK1/Parkin-mediated mitophagy is dependent on VDAC1 and p62/SQSTM1. Nat Cell Biol 12(2):119-131

57. Lazarou $\mathrm{M}$ et al (2015) The ubiquitin kinase PINK1 recruits autophagy receptors to induce mitophagy. Nature 524(7565):309-314

58. Wild P et al (2011) Phosphorylation of the autophagy receptor optineurin restricts Salmonella growth. Science 333(6039):228-233

59. Kachaner D et al (2012) Optineurin: a new vision of cell division control. Cell Cycle 11(8):1481-1482

60. Park B et al (2010) Impairment of protein trafficking upon overexpression and mutation of optineurin. PLoS ONE 5(7):e11547

61. Nagabhushana A, Bansal M, Swarup G (2011) Optineurin is required for CYLD-dependent inhibition of TNFalpha-induced NF-kappaB activation. PLoS ONE 6(3):e17477

62. Heo JM et al (2015) The PINK1-PARKIN mitochondrial ubiquitylation pathway drives a program of OPTN/NDP52 recruitment and TBK1 activation to promote mitophagy. Mol Cell 60(1):7-20

63. Richter B et al (2016) Phosphorylation of OPTN by TBK1 enhances its binding to $\mathrm{Ub}$ chains and promotes selective autophagy of damaged mitochondria. Proc Natl Acad Sci U S A 113(15):4039-4044

64. Bansal M et al (2018) Optineurin promotes autophagosome formation by recruiting the autophagy-related Atg12-5-16L1 complex to phagophores containing the Wipi2 protein. J Biol Chem 293(1):132-147

65. Padman BS et al (2019) LC3/GABARAPs drive ubiquitinindependent recruitment of Optineurin and NDP52 to amplify mitophagy. Nat Commun 10(1):408

66. Marek-Iannucci S et al (2019) Myocardial hypothermia increases autophagic flux, mitochondrial mass and myocardial function after ischemia-reperfusion injury. Sci Rep 9(1):10001

67. McWilliams TG et al (2018) Basal mitophagy occurs independently of PINK1 in mouse tissues of high metabolic demand. Cell Metab 27(2):439-449.e5

68. Kubli DA et al (2013) Parkin protein deficiency exacerbates cardiac injury and reduces survival following myocardial infarction. J Biol Chem 288(2):915-926

69. Billia F et al (2011) PTEN-inducible kinase 1 (PINK1)/Park6 is indispensable for normal heart function. Proc Natl Acad Sci U S A 108(23):9572-9577

70. Tong $\mathrm{M}$ et al (2019) Mitophagy is essential for maintaining cardiac function during high fat diet-induced diabetic cardiomyopathy. Circ Res 124(9):1360-1371

71. Huang $C$ et al (2011) Preconditioning involves selective mitophagy mediated by Parkin and p62/SQSTM1. PLoS ONE 6(6):e20975

72. Andres AM et al (2017) Mitophagy and mitochondrial biogenesis in atrial tissue of patients undergoing heart surgery with cardiopulmonary bypass. JCI Insight 2(4):e89303

73. Woodall BP et al (2019) Parkin does not prevent accelerated cardiac aging in mitochondrial DNA mutator mice. JCI Insight 5:e127713
74. Gong $\mathrm{G}$ et al (2015) Parkin-mediated mitophagy directs perinatal cardiac metabolic maturation in mice. Science $350(6265): \operatorname{aad} 2459$

75. Chen CCW et al (2018) Parkin is required for exercise-induced mitophagy in muscle: impact of aging. Am J Physiol Endocrinol Metab 315(3):E404-E415

76. Liu L et al (2014) Receptor-mediated mitophagy in yeast and mammalian systems. Cell Res 24(7):787-795

77. Zhu Y et al (2013) Modulation of serines 17 and 24 in the LC3interacting region of Bnip3 determines pro-survival mitophagy versus apoptosis. J Biol Chem 288(2):1099-1113

78. Sulistijo ES, Mackenzie KR (2009) Structural basis for dimerization of the BNIP3 transmembrane domain. Biochemistry 48(23):5106-5120

79. Vande Velde C et al (2000) BNIP3 and genetic control of necrosis-like cell death through the mitochondrial permeability transition pore. Mol Cell Biol 20(15):5454-5468

80. Quinsay MN et al (2010) Bnip3 mediates permeabilization of mitochondria and release of cytochrome $\mathrm{c}$ via a novel mechanism. J Mol Cell Cardiol 48(6):1146-1156

81. Zhang H et al (2008) Mitochondrial autophagy is an HIF-1-dependent adaptive metabolic response to hypoxia. J Biol Chem 283(16):10892-10903

82. Bellot $G$ et al (2009) Hypoxia-induced autophagy is mediated through hypoxia-inducible factor induction of BNIP3 and BNIP3L via their BH3 domains. Mol Cell Biol 29(10):2570-2581

83. Quinsay MN et al (2010) Bnip3-mediated mitochondrial autophagy is independent of the mitochondrial permeability transition pore. Autophagy 6(7):855-862

84. Chourasia AH, Macleod KF (2015) Tumor suppressor functions of BNIP3 and mitophagy. Autophagy 11(10):1937-1938

85. Burton TR, Gibson SB (2009) The role of Bcl-2 family member BNIP3 in cell death and disease: NIPping at the heels of cell death. Cell Death Differ 16(4):515-523

86. Quan W, Lee MS (2013) Role of autophagy in the control of body metabolism. Endocrinol Metab (Seoul) 28(1):6-11

87. Sowter HM et al (2003) Expression of the cell death genes BNip3 and NIX in ductal carcinoma in situ of the breast; correlation of BNip3 levels with necrosis and grade. J Pathol 201(4):573-580

88. Okami J, Simeone DM, Logsdon CD (2004) Silencing of the hypoxia-inducible cell death protein BNIP3 in pancreatic cancer. Cancer Res 64(15):5338-5346

89. Chaanine AH et al (2013) Potential role of BNIP3 in cardiac remodeling, myocardial stiffness, and endoplasmic reticulum: mitochondrial calcium homeostasis in diastolic and systolic heart failure. Circ Heart Fail 6(3):572-583

90. Diwan A et al (2007) Inhibition of ischemic cardiomyocyte apoptosis through targeted ablation of Bnip3 restrains postinfarction remodeling in mice. J Clin Invest 117(10):2825-2833

91. Liu L et al (2012) Mitochondrial outer-membrane protein FUNDC1 mediates hypoxia-induced mitophagy in mammalian cells. Nat Cell Biol 14(2):177-185

92. Wu W et al (2016) FUNDC1 regulates mitochondrial dynamics at the ER-mitochondrial contact site under hypoxic conditions. EMBO J 35(13):1368-1384

93. $\mathrm{Wu} \mathrm{W}$ et al (2014) ULK1 translocates to mitochondria and phosphorylates FUNDC1 to regulate mitophagy. EMBO Rep 15(5):566-575

94. Chen $\mathrm{G}$ et al (2014) A regulatory signaling loop comprising the PGAM5 phosphatase and CK2 controls receptor-mediated mitophagy. Mol Cell 54(3):362-377

95. Lampert MA et al (2019) BNIP3L/NIX and FUNDC1mediated mitophagy is required for mitochondrial network 
remodeling during cardiac progenitor cell differentiation. Autophagy 15(7):1182-1198

96. Chen $M$ et al (2016) Mitophagy receptor FUNDC1 regulates mitochondrial dynamics and mitophagy. Autophagy 12(4):689-702

97. Zhou $\mathrm{H}$ et al (2017) Ripk3 induces mitochondrial apoptosis via inhibition of FUNDC1 mitophagy in cardiac IR injury. Redox Biol 13:498-507

98. Yu W et al (2019) Mst1 promotes cardiac ischemia-reperfusion injury by inhibiting the ERK-CREB pathway and repressing FUNDC1-mediated mitophagy. J Physiol Sci 69(1):113-127

99. Zhou $\mathrm{H}$ et al (2018) NR4A1 aggravates the cardiac microvascular ischemia reperfusion injury through suppressing FUNDC1mediated mitophagy and promoting Mff-required mitochondrial fission by CK2alpha. Basic Res Cardiol 113(4):23

100. Saito T et al (2019) An alternative mitophagy pathway mediated by Rab9 protects the heart against ischemia. J Clin Invest 129(2):802-819

101. Thomas A, Marek-Iannucci S, Tucker KC, Andres AM, Gottlieb RA (2020) Decrease of Cardiac Parkin Protein in Obese Mice. Front Cardiovasc Med 6:191. https://doi.org/10.3389/fcvm.2019. 00191

102. Cadenas E, Davies KJA (2000) Mitochondrial free radical generation, oxidative stress, and aging11This article is dedicated to the memory of our dear friend, colleague, and mentor Lars Ernster (1920-1998), in gratitude for all he gave to us. Free Radical Biol Med 29(3):222-230

103. Kukat $\mathrm{C}$ et al (2015) Cross-strand binding of TFAM to a single mtDNA molecule forms the mitochondrial nucleoid. Proc Natl Acad Sci U S A 112(36):11288-11293

104. Marin-Garcia J (2016) Mitochondrial DNA repair: a novel therapeutic target for heart failure. Heart Fail Rev 21(5):475-487

105. Li Y et al (2019) The DNA repair nuclease MRE11A functions as a mitochondrial protector and prevents $\mathrm{T}$ cell pyroptosis and tissue inflammation. Cell Metab 30(3):477-492.e6

106. Ronchi D et al (2019) Novel mutations in DNA2 associated with myopathy and mtDNA instability. Ann Clin Transl Neurol 6(9):1893-1899

107. El-Hattab AW, Craigen WJ, Scaglia F (2017) Mitochondrial DNA maintenance defects. Biochim Biophys Acta Mol Basis Dis 1863(6):1539-1555

108. Mouli PK, Twig G, Shirihai OS (2009) Frequency and selectivity of mitochondrial fusion are key to its quality maintenance function. Biophys J 96(9):3509-3518

109. Glauser L et al (2011) Parkin promotes the ubiquitination and degradation of the mitochondrial fusion factor mitofusin 1 . J Neurochem 118(4):636-645

110. McLelland GL et al (2018) Mfn2 ubiquitination by PINK1/parkin gates the p97-dependent release of ER from mitochondria to drive mitophagy. Elife 7:e32866

111. Buchberger A, Bukau B, Sommer T (2010) Protein quality control in the cytosol and the endoplasmic reticulum: brothers in arms. Mol Cell 40(2):238-252

112. Mayer MP, Bukau B (2005) Hsp70 chaperones: cellular functions and molecular mechanism. Cell Mol Life Sci 62(6):670-684

113. Cox JS, Chapman RE, Walter P (1997) The unfolded protein response coordinates the production of endoplasmic reticulum protein and endoplasmic reticulum membrane. Mol Biol Cell 8(9):1805-1814
114. Hollien J, Weissman JS (2006) Decay of endoplasmic reticulumlocalized mRNAs during the unfolded protein response. Science 313(5783):104-107

115. Houtkooper RH et al (2013) Mitonuclear protein imbalance as a conserved longevity mechanism. Nature 497(7450):451-457

116. Liu Y et al (2014) Caenorhabditis elegans pathways that surveil and defend mitochondria. Nature 508(7496):406-410

117. Nargund AM et al (2012) Mitochondrial import efficiency of ATFS-1 regulates mitochondrial UPR activation. Science 337(6094):587-590

118. Haynes CM et al (2010) The matrix peptide exporter HAF-1 signals a mitochondrial UPR by activating the transcription factor ZC376.7 in C.elegans. Mol Cell 37(4):529-540

119. Zhao $Q$ et al (2002) A mitochondrial specific stress response in mammalian cells. EMBO J 21(17):4411-4419

120. Munch C, Harper JW (2016) Mitochondrial unfolded protein response controls matrix pre-RNA processing and translation. Nature 534(7609):710-713

121. Fiorese CJ et al (2016) The transcription factor ATF5 mediates a mammalian mitochondrial UPR. Curr Biol 26(15):2037-2043

122. Nargund AM et al (2015) Mitochondrial and nuclear accumulation of the transcription factor ATFS-1 promotes OXPHOS recovery during the UPR(mt). Mol Cell 58(1):123-133

123. Munch $C$ (2018) The different axes of the mammalian mitochondrial unfolded protein response. BMC Biol 16(1):81

124. Radke $\mathrm{S}$ et al (2008) Mitochondrial protein quality control by the proteasome involves ubiquitination and the protease Omi. J Biol Chem 283(19):12681-12685

125. Papa L, Germain D (2011) Estrogen receptor mediates a distinct mitochondrial unfolded protein response. J Cell Sci 124(Pt 9):1396-1402

126. Riar AK et al (2017) Sex specific activation of the ERalpha axis of the mitochondrial UPR (UPRmt) in the G93A-SOD1 mouse model of familial ALS. Hum Mol Genet 26(7):1318-1327

127. Kirstein $\mathrm{J}$ et al (2009) Adapting the machine: adaptor proteins for Hsp100/Clp and AAA+ proteases. Nat Rev Microbiol 7(8):589-599

128. Sauer RT, Baker TA (2011) AAA+ proteases: ATP-fueled machines of protein destruction. Annu Rev Biochem 80:587-612

129. Bezawork-Geleta A et al (2015) LON is the master protease that protects against protein aggregation in human mitochondria through direct degradation of misfolded proteins. Sci Rep 5:17397

130. Lebeau J et al (2018) The PERK arm of the unfolded protein response regulates mitochondrial morphology during acute endoplasmic reticulum stress. Cell Rep 22(11):2827-2836

131. Onat UI et al (2019) Intercepting the lipid-induced integrated stress response reduces atherosclerosis. J Am Coll Cardiol 73(10):1149-1169

132. Picca A et al (2018) Mitochondrial quality control mechanisms as molecular targets in cardiac ageing. Nat Rev Cardiol 15(9):543-554

Publisher's Note Springer Nature remains neutral with regard to jurisdictional claims in published maps and institutional affiliations. 